\title{
Severity of pancreatitis-associated intestinal mucosal barrier injury is reduced following treatment with the NADPH oxidase inhibitor apocynin
}

\author{
WENHONG DENG ${ }^{1}$, ABLIKIM ABLIZ ${ }^{1}$, SHENG XU ${ }^{2}$, RONGZE SUN $^{1}$, \\ WENYI GUO ${ }^{1}$, QIAO SHI ${ }^{1}$, JIA YU ${ }^{1}$ and WEIXING WANG ${ }^{1}$ \\ ${ }^{1}$ Department of General Surgery, Renmin Hospital of Wuhan University, Wuhan, Hubei 430060; \\ ${ }^{2}$ Department of General Surgery, People's Hospital of Guangxi Zhuang Autonomous Region, \\ Nanning, Guangxi 530021, P.R. China
}

Received June 4, 2015; Accepted June 17, 2016

DOI: $10.3892 / \mathrm{mmr} .2016 .5678$

\begin{abstract}
Recent studies demonstrated that apocynin, a nicotinamide adenine dinucleotide phosphate (NADPH) oxidase (NOX) inhibitor, significantly decreased acute pancreatitis-associated inflammatory and oxidative stress parameters. In addition, apocynin was able to reduce ischemic reperfusion injury-associated damage; however, the exact effects of apocynin on acute pancreatitis-associated intestinal mucosal injury have yet to be fully clarified. The present study aimed to investigate the protective effects of apocynin on intestinal mucosal injury in a rat model of severe acute pancreatitis (SAP). A total of 60 male Sprague Dawley rats were randomly divided into four groups (n=15/group): Sham operation group (SO), SAP group, apocynin treatment (APO) group and drug control (APO-CON) group. SAP was induced by retrograde injection of $5 \%$ sodium taurocholate into the biliopancreatic duct. Apocynin was administered 30 min prior to SAP induction in the APO group. All rats were sacrificed $12 \mathrm{~h}$ after SAP induction. Intestinal integrity was assessed by measuring diamine oxidase (DAO) levels. Morphological alterations to intestinal tissue were determined under light and transmission electron microscopy. NOX2, p38 mitogen-activated protein kinases (MAPK) and nuclear factor $(\mathrm{NF})-\mathrm{\kappa B}$ expression levels were detected in
\end{abstract}

Correspondence to: Professor Weixing Wang, Department of General Surgery, Renmin Hospital of Wuhan University, 238 Jiefang Road, Wuhan, Hubei 430060, P.R. China

E-mail: sate.1lite@163.com

Dr Sheng Xu, Department of General Surgery, People's Hospital of Guangxi Zhuang Autonomous Region, 6 Taoyuan Road, Nanning, Guangxi 530021, P.R. China

E-mail: xvsheng@hotmail.com

Key words: severe acute pancreatitis, intestinal mucosal injury, NADPH oxidase, apocynin, p38 mitogen-activated protein kinases, nuclear factor- $\kappa \mathrm{B}$ the intestine by immunohistochemical staining. Oxidative stress was detected by measuring intestinal malondialdehyde (MDA) and superoxide dismutase content. In addition, blood inflammatory cytokines, and amylase (AMY) and lipase (LIP) levels were evaluated. The results demonstrated that apocynin attenuated the following: i) Serum AMY, LIP and DAO levels; ii) pancreatic and intestinal pathological injury; iii) intestinal MDA content; iv) intestinal ultrastructural alterations; v) serum interleukin (IL)-1 $\beta$, IL-6 and tumor necrosis factor (TNF)- $\alpha$ levels; and vi) NOX2, p38 MAPK and $\mathrm{NF}-\mathrm{\kappa B}$ expression in intestinal tissues. These results suggested that apocynin may attenuate intestinal barrier dysfunction in sodium taurocholate-induced SAP, presumably via its role in the prevention of reactive oxygen species generation and inhibition of $\mathrm{p} 38$ MAPK and NF- $\mathrm{kB}$ pathway activation. These findings provide novel insight suggesting that pharmacological inhibition of NOX by apocynin may be considered a novel therapeutic method for the treatment of intestinal injury in SAP.

\section{Introduction}

Severe acute pancreatitis (SAP) is a common acute abdominal disease, which can lead to early mortality of patients due to associated systemic inflammatory response syndrome (SIRS) and multiple organ dysfunction syndrome (MODS) (1). SAP-associated failure of the gastrointestinal tract, which is characterized by endotoxin release from the intestinal lumen, and translocation of enteric bacteria to extraintestinal sites and systemic circulation, leads to increased intestinal permeability and release of inflammatory mediators and cytokines, which has a pivotal role during SAP. Bacteremia, infected necrosis, organ failure and mortality are all associated with intestinal barrier dysfunction in the early stages of SAP (2).

The mechanisms underlying SAP-induced intestinal mucosal injury have yet to be fully elucidated. Previous studies have suggested that intestinal mucosal injury is a complex pathophysiological process that involves several factors, including inflammatory mediators, oxidative stress and intestinal hypoperfusion $(3,4)$. Therefore, it is clinically imperative 
to study the association between injury of the intestinal mucosal barrier and SAP. In addition, microcirculation disturbance, ischemic reperfusion injury, excessive release of inflammatory mediators and apoptosis may also have important roles in damaging the intestinal mucosal barrier (5). Microcirculation disturbance results in gut barrier dysfunction via the production of reactive oxygen species (ROS) by xanthine oxidase (XO), and hypoxanthine accumulation in intestinal tissue (6). It has previously been reported that increased ROS generation and a dysregulated antioxidant defense system in intestinal mucosal cells is involved in intestinal mucosal dysfunction in rats with SAP (6).

Nicotinamide adenine dinucleotide phosphate (NADPH) oxidase (NOX) is considered a potential source of oxidants in the injured intestine in SAP. NOX produces a large amount of ROS predominantly in professional phagocytes, such as neutrophils and macrophages (7). NOX is a multicomponent enzyme; the classical phagocytic NOX is comprised of the membrane-bound subunits gp22phox and gp91phox (also termed NOX2), as well as the cytosolic subunits, including gp67phox and gp47phox (7). NOX has a crucial role in various biological processes, including host defense, signal transduction, mitogenic growth, hormone synthesis, apoptosis, angiogenesis, and oxidative modification of the extracellular matrix and extracellular proteins (8). In addition, NOX is involved in various types of disease, including atherosclerosis, cancer, diabetes, defective immune function and inflammatory conditions, such as inflammatory bowel disease (8). Previous studies have demonstrated that NOX is involved in mitogen-activated protein kinases (MAPK), phospholipase A2 and nuclear factor (NF) $-\kappa \mathrm{B}$ activation, and cytokine expression, and therefore, pathogenesis of pancreatitis $(9,10)$. Experimental studies have suggested that the source of ROS is NOX in stimulated neutrophils in cerulein pancreatitis in vivo (11). Therefore, treatments designed to modulate the production of ROS by NOX enzymes may provide a novel therapeutic approach for the treatment of some of these conditions.

Apocynin is a selective NOX inhibitor, which exhibits low toxicity, and may therefore be considered a promising potential therapy for asthma, arthritis, and neurological and cardiovascular diseases via its antioxidant and anti-inflammatory effects. In addition, apocynin has been used in several experimental studies associated with ischemic reperfusion injury $(12,13)$.

At present, the protective effects of apocynin on the intestinal mucosal barrier in rats with SAP have yet to be investigated. The present study hypothesized that NOX is involved in the pathogenesis of SAP-associated acute intestinal injury, and aimed to evaluate the effects of the NOX inhibitor apocynin on SAP-associated intestinal mucosal injury. The investigation of the effects of apocynin on SAP-associated intestinal injury may provide a novel basis for the treatment of SAP.

\section{Materials and methods}

Animals. A total of 60 male adult Sprague Dawley rats (age, 7-8 weeks; weight, 200-250 g) were obtained from Hubei Experimental Animal Center (Wuhan, China). The rats were housed in a climate-controlled room with an ambient temperature of $23^{\circ} \mathrm{C}$ and were maintained under a $12: 12 \mathrm{~h}$ light-dark cycle. The rats were fed standard laboratory chow, given ad libitum access to water, and were randomly assigned to four groups ( $\mathrm{n}=15 /$ group): Sham operation group (SO), SAP group, apocynin treatment (APO) group and drug control (APO-CON) group. All animal study procedures complied with international guidelines for the care and use of laboratory animals, and were approved by the Animal Ethics Committee of Wuhan University (Wuhan, China).

SAP induction and sample collection. The rats were fasted $12 \mathrm{~h}$ prior to the experiment, however drinking water remained available. The SAP model was induced by a standardized pressure-controlled retrograde infusion of 5\% sodium taurocholate $(1 \mathrm{ml} / \mathrm{kg})$ into the biliopancreatic duct. In the SO and APO-CON groups, an incision was made in the abdomen of the rats under chloral hydrate $(10 \%, 30 \mathrm{mg} / \mathrm{kg}$; Aoxin Chemical Factory, Yangzhou, China) anesthesia and was subsequently closed. Following the operation, all rats received subcutaneous infusion of sterile saline $(2 \mathrm{ml} / \mathrm{kg}$ ) to compensate for anticipated fluid loss. In the APO group, 10\% dimethyl sulfoxide (DMSO) containing apocynin $(50 \mathrm{mg} / \mathrm{kg}$; Selleck Chemicals, Houston, TX, USA) was injected very slowly through the femoral vein $30 \mathrm{~min}$ prior to SAP induction. In the SO and SAP groups, $10 \%$ DMSO solution $(2 \mathrm{ml} / \mathrm{kg}$ ) was administered via femoral vein $30 \mathrm{~min}$ prior to the operation. In the APO-CON group, 10\% DMSO containing apocynin $(50 \mathrm{mg} / \mathrm{kg}$ ) was injected very slowly through the femoral vein 30 min prior to the sham operation.

A total of $12 \mathrm{~h}$ after SAP induction, rats from each group were anesthetized with $10 \%$ chloral hydrate $(30 \mathrm{mg} / \mathrm{kg})$, the abdomen was opened, and pancreatic and ileum tissues adjacent to the cecum were rapidly collected. All rats were sacrificed by the collection of blood samples $(5 \mathrm{ml})$ were from the left ventricle of the heart. The samples were centrifuged at $300 \mathrm{x} \mathrm{g}$ for $15 \mathrm{~min}$ at $4^{\circ} \mathrm{C}$, and the serum was stored at $-20^{\circ} \mathrm{C}$ until further analysis. Half of each pancreatic and ileal sample was fixed with $40 \mathrm{~g} / 1$ formaldehyde, embedded in paraffin and cut into $5 \mathrm{~mm}$ sections. The other half was stored at $-80^{\circ} \mathrm{C}$ for further use.

Biochemical assays. The serum levels of amylase (AMY) and lipase (LIP) were measured according to the manufacturer's instructions, using an automatic biochemistry analyzer (model AU600; Olympus Corporation, Tokyo, Japan). Serum tumornecrosis factor(TNF)- $\alpha$ (cat.no. BP-E40017), interleukin (IL)-6 (cat. no. BP-E30419) and IL-1 $\beta$ (cat. no. BP-E30419) levels were determined using enzyme-linked immunosorbent assay (ELISA) kits (Biomart, Co., Toronto, Canada) according to the manufacturer's protocols. For diamine oxidase (DAO) detection, a rat DAO ELISA kit (cat. no. RA20156; Shanghai DaWeiKe Biotechnology, Shanghai, China) was used according to the manufacturer's protocol. Tissue malondialdehyde (MDA), a marker of lipid peroxidation, and superoxide dismutase (SOD) levels were measured using commercial MDA (cat. no. A003-1) and SOD (cat. no. A001-1) assay kits (Nanjing Jiancheng Bioengineering Institute, Nanjing, China) according to the manufacturer's instructions.

Histopathological evaluation. Paraffin-embedded samples from the pancreas and ileum were sectioned $(5 \mu \mathrm{m})$, stained with hematoxylin and eosin for $10 \mathrm{~min}$ at room temperature, and examined by an experienced pathologist who was blinded 

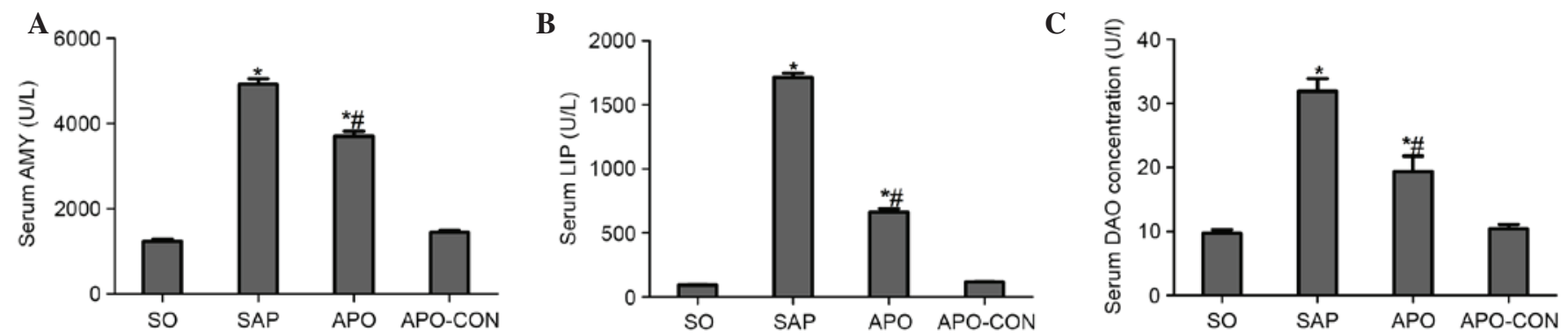

Figure 1. Serum levels of amylase (AMY), lipase (LIP) and diamine oxidase (DAO) in all rat groups. (A) Serum AMY levels, (B) serum LIP levels and (C) serum DAO levels. Results are presented as the mean \pm standard deviation from 15 rats per group. ${ }^{*} \mathrm{P}<0.05$ vs. sham operation $(\mathrm{SO})$ group; ${ }^{*} \mathrm{P}<0.05$ vs. severe acute pancreatitis (SAP) group. APO, apocynin group; APO-CON, drug control group.

to the sample identity. Histopathological alterations to the pancreas were evaluated according to the criteria proposed by Schmidt et al (14). The histological grade of intestinal mucosal damage was scored according to the standard scale described by Chiu et al (15). Briefly, mucosal damage was graded from 0 to 5 according to the following criteria: Grade 0 , normal mucosal villi; grade 1, development of subepithelial Gruenhagen's space at the apex of the villus, often with capillary congestion; grade 2, extension of the subepithelial space with moderate lifting of the epithelial layer from the lamina propria; grade 3, massive epithelial lifting down the sides of villi, possibly with a few denuded tips; grade 4, denuded villi with the lamina propria and dilated capillaries exposed, possibly with increased cellularity of the lamina propria; and grade 5, digestion and disintegration of the lamina propria, hemorrhage and ulceration.

Immunohistochemistry. NOX2, p38 MAPK and $\mathrm{NF}-\kappa \mathrm{B}$ protein expression was detected in the intestinal mucosa by immunohistochemistry. The paraffin-embedded ileal tissue sections were dewaxed according to standard procedures (16). Subsequently, the sections were dipped in a solution containing $0.01-\mathrm{mol} / \mathrm{l}$ citric acid for $1 \mathrm{~min}$ at $95^{\circ} \mathrm{C}$, blocked with $5 \%$ bovine serum (Jingmei BioTech Co., Ltd., Yencheng, China) for $30 \mathrm{~min}$, and were then incubated with rabbit anti-p38 (1:50; cat. no. sc-535), anti-phosphorylated (p)-P38 (1:50; cat. no. sc-101759), anti-NF- $\mathrm{B}$ (1:50; cat. no. sc-8008) and anti-NOX2 (1:100; cat. no. sc-6706) polyclonal antibodies (Santa Cruz Biotechnology, Inc., Dallas, TX, USA) overnight at $4^{\circ} \mathrm{C}$. Sections were then incubated with biotin-labeled goat anti-rabbit immunoglobulin G (1:200; cat. no. sc-2012; Santa Cruz Biotechnology, Inc.) for $15 \mathrm{~min}$ at $37^{\circ} \mathrm{C}$, and horseradish peroxidase-labeled streptavidin (Wuhan Feiyi Technology Co., Ltd., Wuhan, China) for $30 \mathrm{~min}$ at $37^{\circ} \mathrm{C}$. Finally, the specimens were stained with diaminobenzidine, nuclear counter-stained with hematoxylin, and images were captured under a Leica DM 2500 microscope (Leica Microsystems GmbH, Wetzlar, Germany). Brown staining area represents cells that contain p38, p-p38, NF- $\mathrm{B}$ and NOX2. The positively stained cells were observed under a light microscope (magnification, 400x) and were evaluated by two pathologists in a blind manner.

Immunohistochemical staining was analyzed using Image Pro-Plus (version 6.0; Media Cybernetics, Inc., Rockville, MD, USA). Briefly, the positive staining area was selected as the area of interest (AOI), and the area sum and integrated optical density (IOD) of the AOI were selected as measurement parameters. The target protein expression index equaled the quotient between the IOD and the total AOI. Finally, statistical analysis of the mean expression index for each duplicate was performed.

Transmission electron microscopy (TEM). Fresh ileal tissue samples were fixed in a mixture of $2 \%$ formaldehyde and $2 \%$ glutaraldehyde in $0.1 \mathrm{~mol} / 1$ cacodylate buffer $(\mathrm{pH} 7.4)$ overnight. The samples were postfixed in $1 \% \mathrm{OsO}_{4}$ in the same buffer, en bloc-stained with $2 \%$ aqueous uranyl acetate, dehydrated in ethanol, and embedded in Poly/Bed 812 (Polysciences, Inc., Warrington, PA, USA). Ultrathin sections $(50 \mathrm{~nm})$ were cut on a Leica EMUC7 ultramicrotome (Leica Microsystems $\mathrm{GmbH}$ ), stained with lead citrate, and alterations to the intestinal epithelial cells and microvilli were examined under a JEM-2010F electron microscope.

Statistical analysis. Statistical analyses were carried out using SPSS statistical software (PASW Statistics for Windows, version 18.0; SPSS Inc., Chicago, IL, USA). Data are expressed as the mean \pm standard deviation. One-way analysis of variance and least-significant difference test was used to investigate differences among the experimental groups. $\mathrm{P}<0.05$ was considered to indicate a statistically significant difference.

\section{Results}

Effects of NOX inhibition on pancreatic injury and intestinal mucosal dysfunction in SAP. As shown in Fig. 1, significant hyperamylasemia and hyperlipasemia developed following induction of SAP, thus suggesting that the SAP model was successfully induced. Treatment with apocynin reduced AMY and LIP levels in the APO group compared with the SAP group $(\mathrm{P}<0.05$; Fig. $1 \mathrm{~A}$ and $\mathrm{B})$. In addition, rats subjected to SAP exhibited a significant increase in DAO levels $(\mathrm{P}<0.05)$, indicating that the rats experienced aggravated intestinal dysfunction. Apocynin treatment resulted in a significant decrease in the serum levels of DAO $12 \mathrm{~h}$ after SAP induction $(\mathrm{P}<0.05$; Fig. 1C).

Inhibition of NOX attenuates pancreatic and intestinal histopathology. Representative histological sections are presented in Figs. 2 (pancreas) and 3 (intestinal tissue). No 

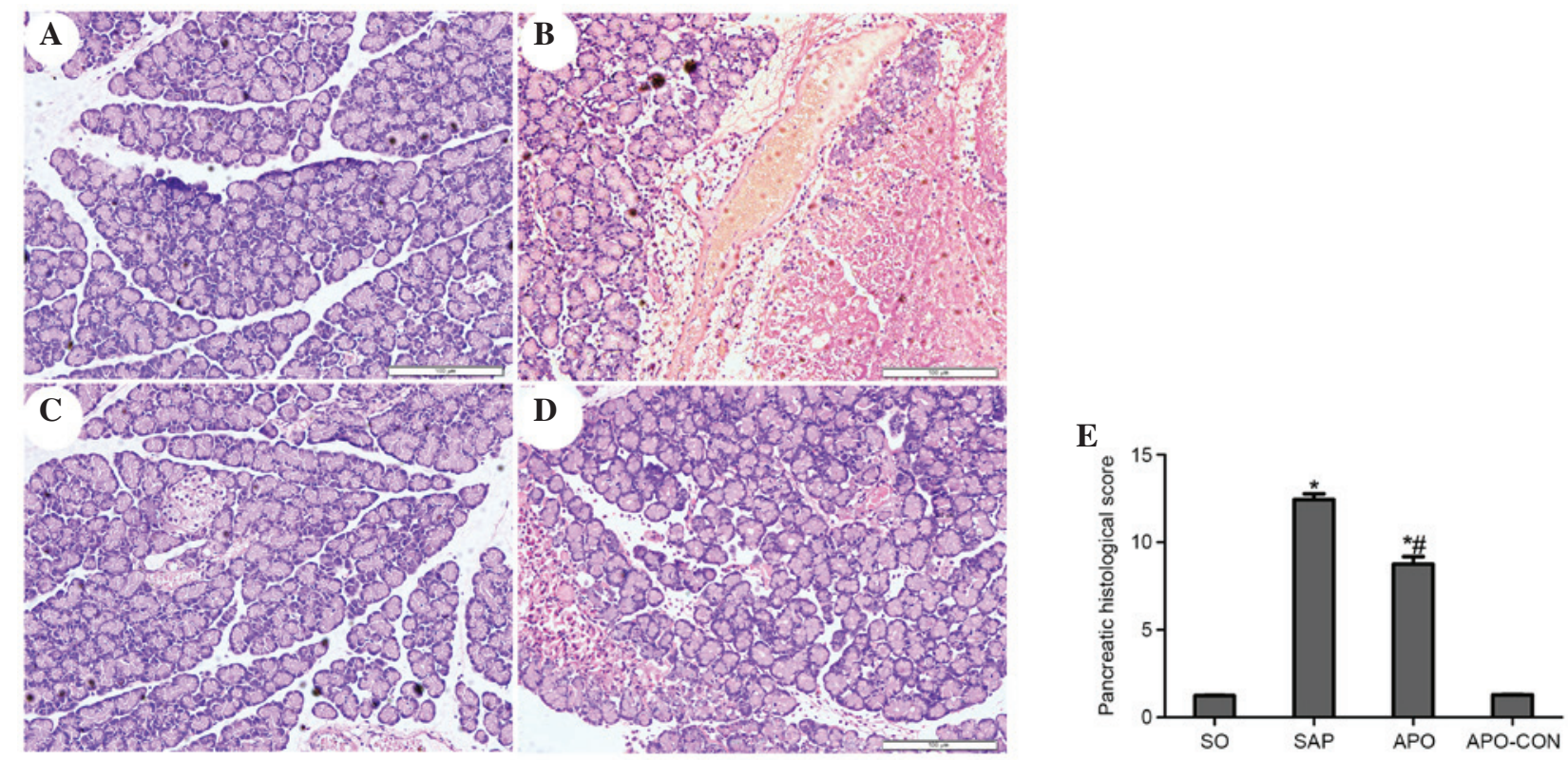

Figure 2. Treatment of rats with apocynin attenuated pancreatic morphological alterations. Representative images from the (A) sham operation (SO) group; (B) severe acute pancreatitis (SAP) group; (C) drug control (APO-CON) group; and (D) apocynin (APO) group (original magnification, x200). (E) Pancreatic histological scores. Results are presented as the mean \pm standard deviation. ${ }^{*} \mathrm{P}<0.05$ vs. SO group; ${ }^{\text {}} \mathrm{P}<0.05$ vs. SAP group.
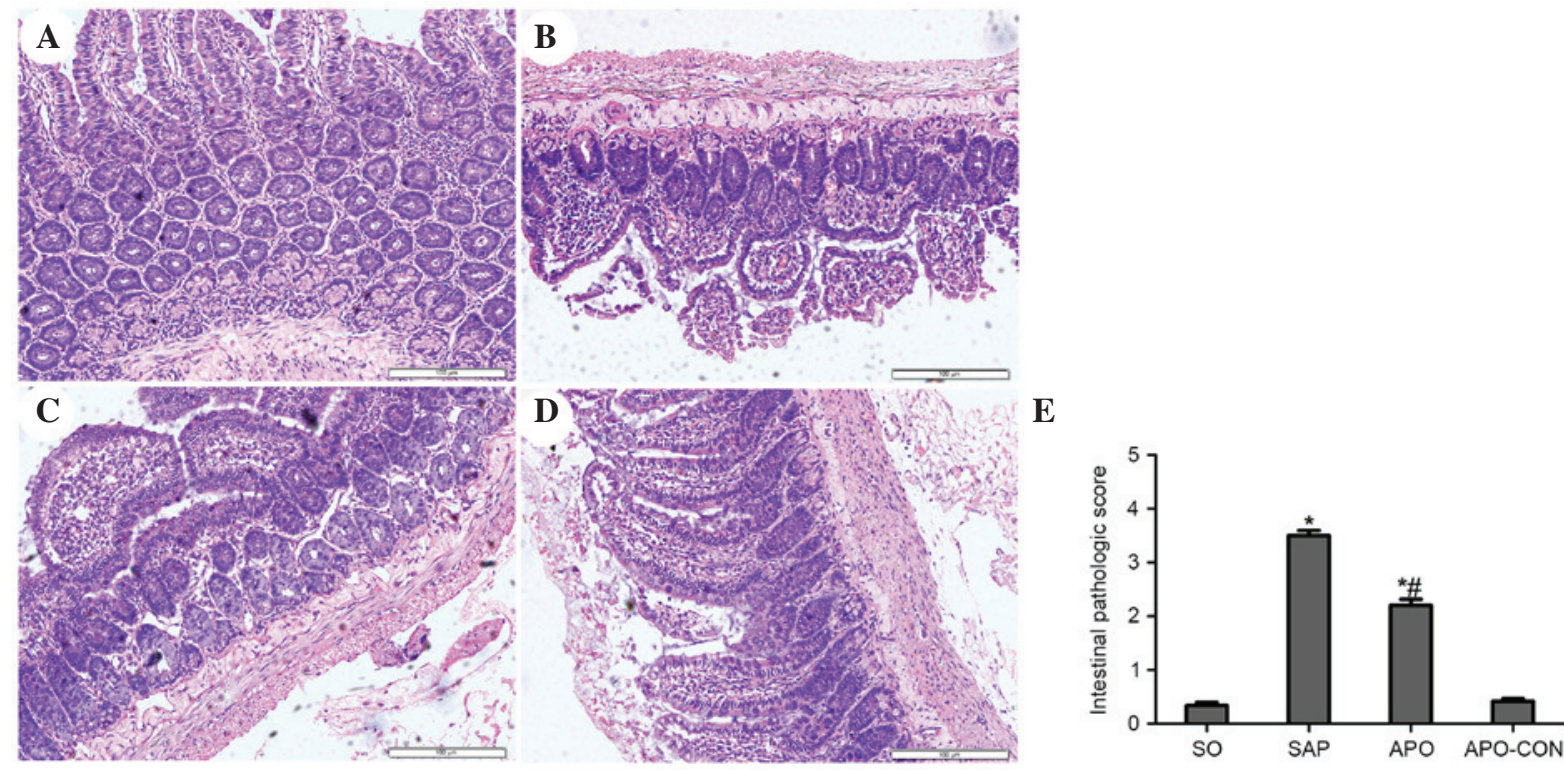

Figure 3. Treatment of rats with apocynin attenuated intestinal morphological alterations. Representative images from the (A) sham operation (SO) group; (B) severe acute pancreatitis (SAP) group; (C) drug control (APO-CON) group; and (D) apocynin (APO) group (original magnification, x200). (E) Intestinal histological scores. Results are presented as the mean \pm standard deviation. ${ }^{\mathrm{P}}<0.05$ vs. SO group; ${ }^{\mathrm{P}}<0.05$ vs. SAP group.

morphological alterations were observed in the SO and APO-CON groups. Interstitial edema, acinar cell necrosis and infiltration of inflammatory cells were observed in the SAP group (Fig. 2). Treatment with APO markedly decreased pancreatic histological injuries $(\mathrm{P}<0.05$; Fig. 2). The $\mathrm{SO}$ and APO-CON groups exhibited normal histological structure of intestinal mucosal tissue (Fig. 3). Conversely, characteristic signs of intestinal injuries, including edema, villose exfoliation, degeneration of mucosal cells, mucosal cell necrosis, bleeding, and leukocytic infiltration were observed in the SAP group (Fig. 3). Intestinal issues obtained from the rats treated with apocynin were shown to have milder histological features and lower pathological scores compared with the SAP group $(\mathrm{P}<0.05)$ (Fig. 3).

Apocynin treatment reduces oxidative stress and production of proinflammatory cytokines. Intestinal tissue MDA levels were significantly elevated in the SAP group compared with in the SO group $(\mathrm{P}<0.05$; Fig. $4 \mathrm{~A})$. Conversely, the elevation was significantly inhibited by apocynin treatment $(\mathrm{P}<0.05$; Fig. 4A). SOD activity was significantly depleted in the SAP group, probably as a result of oxidative stress. Conversely, treatment with apocynin effectively improved SOD activity in intestinal tissues $(\mathrm{P}<0.05$; Fig. 4B). 


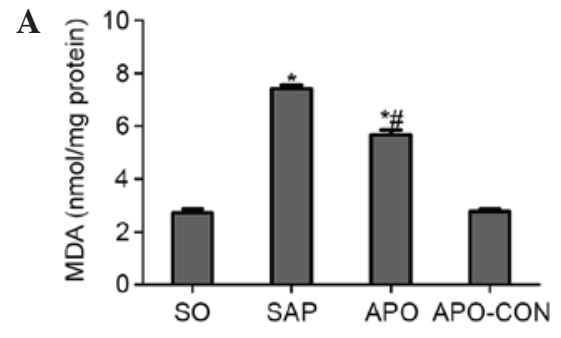

D
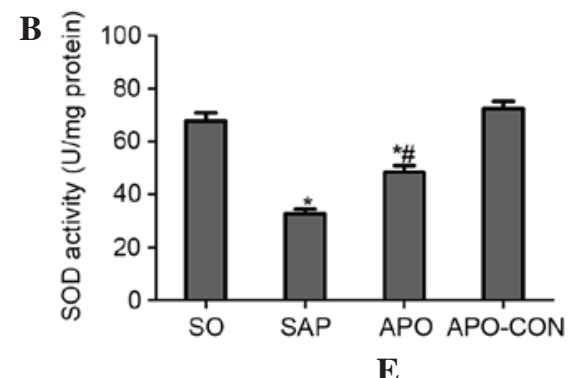

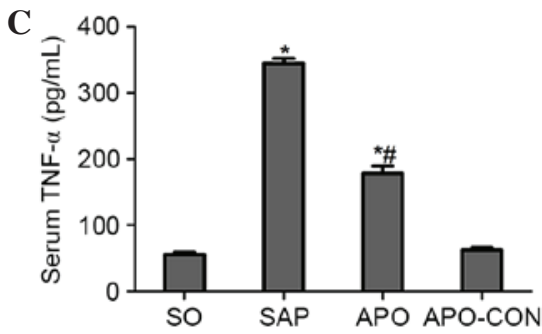

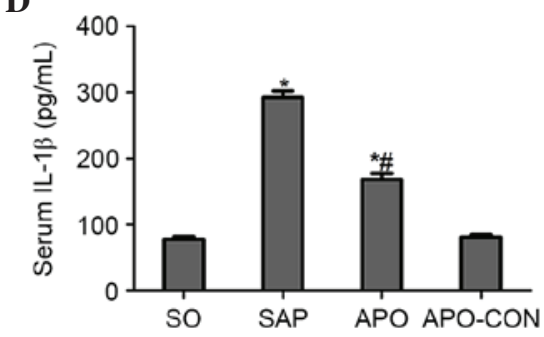

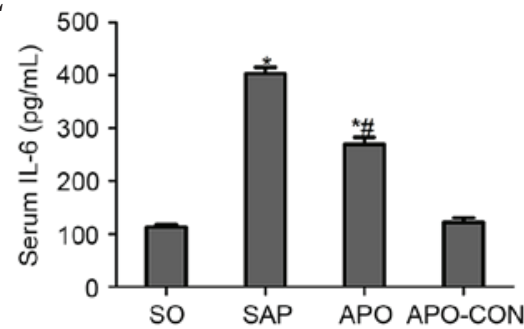

Figure 4. Effects of apocynin on oxidative stress and proinflammatory cytokine production. (A) Malondialdehyde (MDA) content; (B) superoxide dismutsase (SOD) activity; and (C) tumor necrosis factor (TNF)- $\alpha$, (D) interleukin (IL)-1 $\beta$ and (E) IL-6 levels. Results are presented as the mean \pm standard deviation from 15 rats per group. ${ }^{*} \mathrm{P}<0.05$ vs. sham operation (SO) group; ${ }^{*} \mathrm{P}<0.05$ vs. severe acute pancreatitis (SAP) group. APO, apocynin group; APO-CON, drug control group.

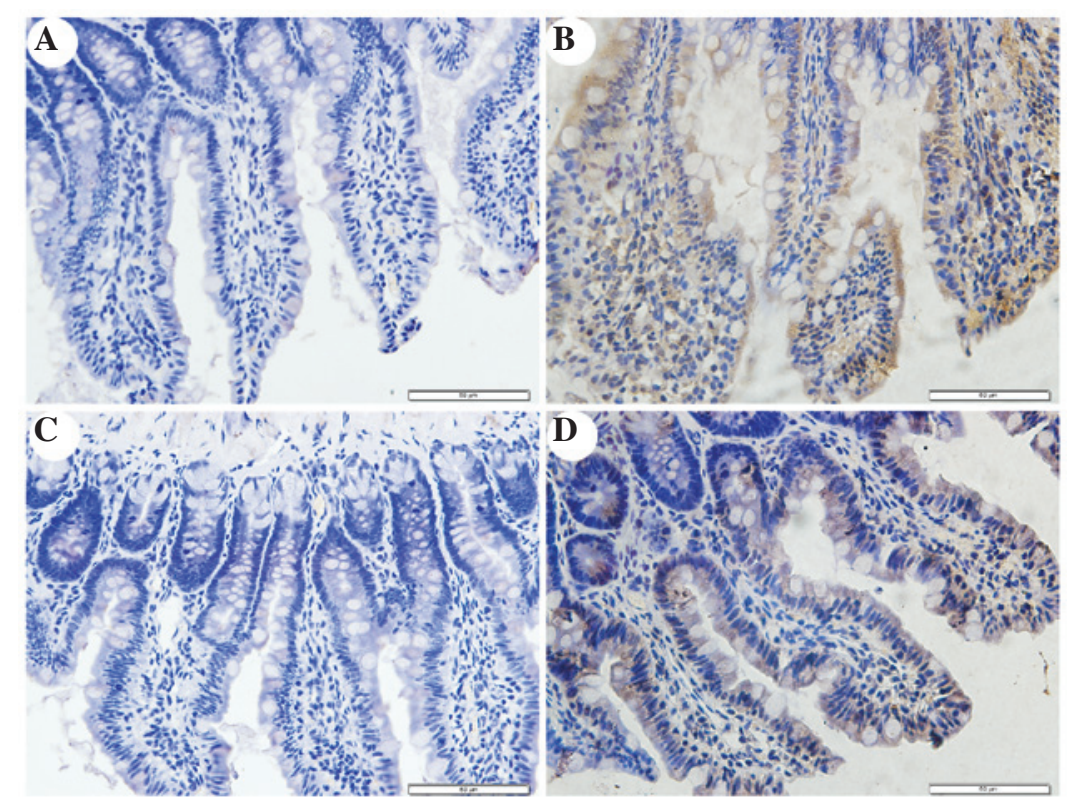

Figure 5. Nicotinamide adenine dinucleotide phosphate oxidase 2 (NOX2) immunohistochemical staining in rat intestinal tissue. Immunohistochemical localization of NOX2 appears as brown staining. (A) Sham operation (SO) group; (B) severe acute pancreatitis (SAP) group; (C) drug control (APO-CON) group; and (D) apocynin (APO) group (original magnification, $\mathrm{x} 400$ ).

Serum concentrations of proinflammatory cytokines were analyzed in order to evaluate the effects of NOX inhibition on inflammatory process following SAP. As presented in Fig. 4, concomitant with the pathological damage incited by SAP, serum levels of TNF- $\alpha$, IL- $1 \beta$ and IL- 6 were significantly increased compared with in the SO group $(\mathrm{P}<0.05$; Fig. $4 \mathrm{C}-\mathrm{E})$. However, the increased levels of these cytokines following SAP induction were markedly decreased by the pharmacological blockade of NOX, using the NOX inhibitor apocynin (P<0.05; Fig. 4C-E).

Inhibition of NOX reduces the expression of $p 38$ MAPK and $N F-\kappa B$ in intestinal mucosa after SAP. Immunohistochemical staining of NOX2 was used to evaluate whether apocynin was able to successfully attenuate SAP-induced NOX2 expression in intestinal tissue (Fig. 5). Intestinal tissue sections from the $\mathrm{SO}$ and APO-CON rats exhibited little NOX2 expression (Fig. 5A and C). However, NOX2 immunoreactivity was enhanced in SAP rats (Fig. 5B) and was reduced in the intestinal tissue of rats that had undergone apocynin pretreatment (Fig. 5D).

The present study determined the efficacy of apocynin treatment on NF- $\mathrm{BB}$, p38 MAPK and p-p38 MAPK expression in intestinal mucosa using immunohistochemical assays

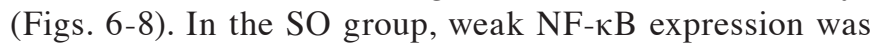
detected in the cytoplasm (Fig. 6A), and the expression of p38 MAPK and p-p38 MAPK in the cytoplasm was 


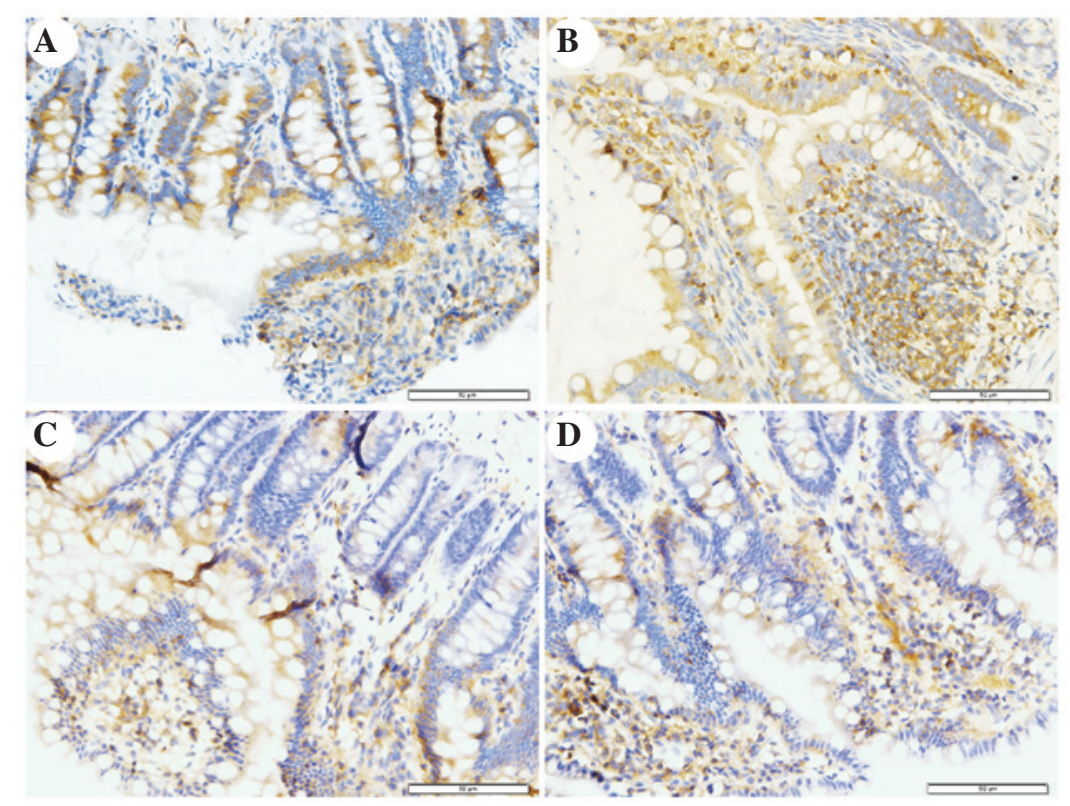

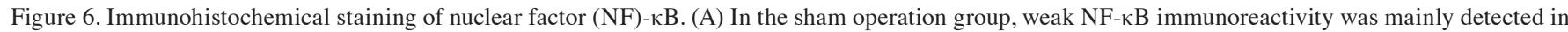
the cytoplasm. (B) In the severe acute pancreatitis (SAP) group, intense NF- $\mathrm{KB}$ immunoreactivity was detected in the nucleus. (C) In the drug control group,

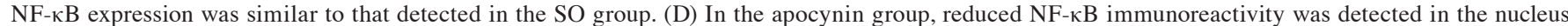
compared with in the SAP group (original magnification, $\mathrm{x} 400$ ).
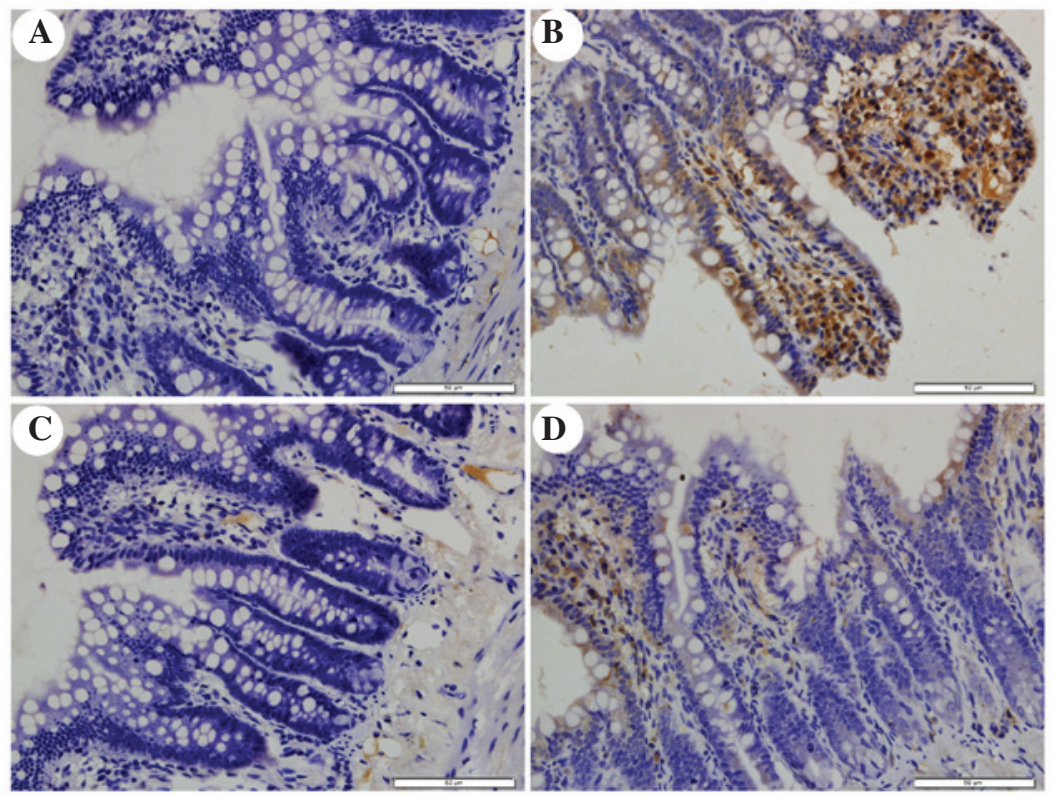

Figure 7. Immunohistochemical staining of p38 mitogen-activated protein kinases (MAPK). (A) In the sham operation group, exceedingly weak p38 MAPK immunoreactivity was detected in the cytoplasm. (B) In the severe acute pancreatitis (SAP) group, intense p38 MAPK immunoreactivity was detected in the cytoplastm and some nuclei. (C) In the drug control group, weak p38 MAPK expression was detected. (D) In the apocynin group, reduced p38 MAPK immunoreactivity was detected in the cytoplasm compared with in the SAP group (original magnification, $\mathrm{x} 400$ ).

exceedingly weak (Figs. 7A and 8A). In the SAP group, intense NF- $\mathrm{kB}$ p 65 immunoreactivity was expressed in the nucleus (Fig. 6B). Intestinal expression of p38 MAPK and p-p38 MAPK was significantly increased in the cytoplasm of the SAP group compared with in the SO group (Figs. 7B and $8 \mathrm{~B} ; \mathrm{P}<0.05)$. In addition, inhibition of NOX with apocynin significantly decreased the nuclear expression of NF- $\mathrm{kB}$ and cytoplasmic expression of p38 MAPK in intestinal tissues (Fig. 9; $\mathrm{P}<0.05$ ).
Ultrastructural alterations to intestinal mucosal cells, as detected under TEM. TEM analysis of intestinal mucosa demonstrated that integrity of the intestinal villi and intestinal epithelial cells (IECs) of the rats in the SO group was maintained throughout the experiment. The mitochondria, endoplasmic reticulum, ribosomes and other cellular organelles were normal following examination at a magnification of x20,000 (Fig. 10A and B). The microvilli and IECs in the SAP group exhibited a loss of integrity. Microvillus atrophy, enlarged 

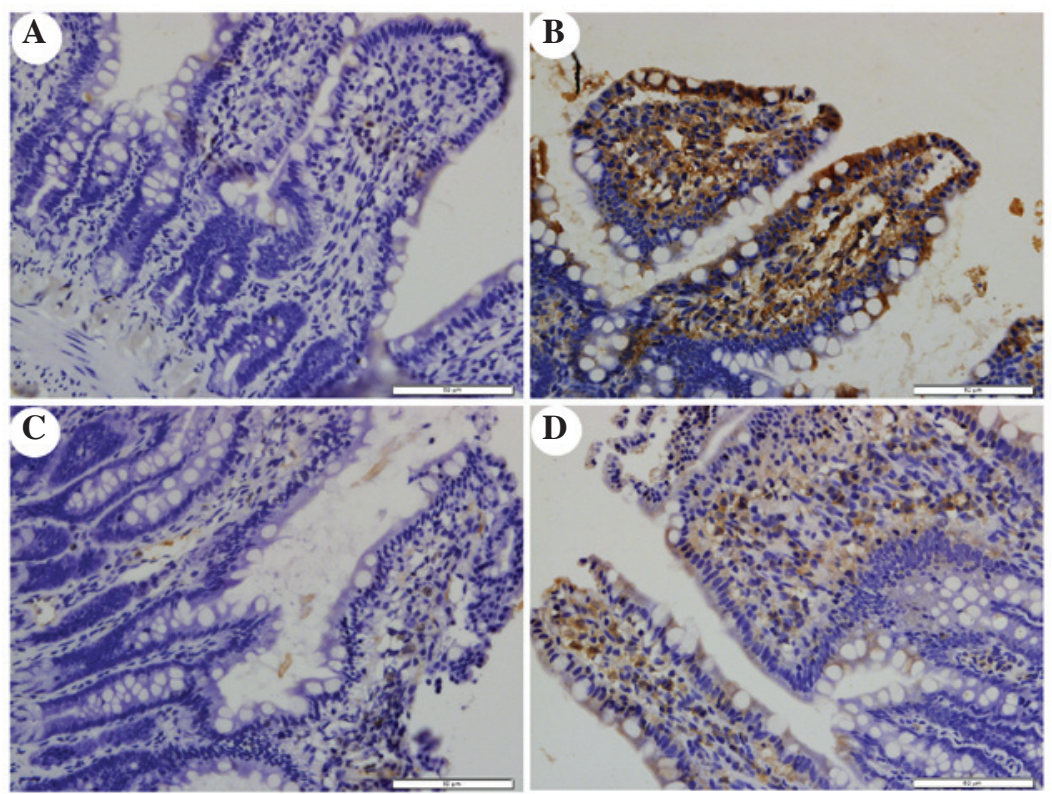

Figure 8. Immunohistochemical staining of phosphorylated (p)-p38 mitogen-activated protein kinases (MAPK). (A) Weak p-p38 MAPK expression was detected in the sham operation group (B). In the severe acute pancreatitis group, p-p38 MAPK immunostaining was increased in cytoplasm and nuclei. (C) In the drug control group, weak p-p38 MAPK expression was detected. (D) Immunostaining was significantly decreased in the apocynin-treated group (original magnification, $\mathrm{x} 400)$.

A

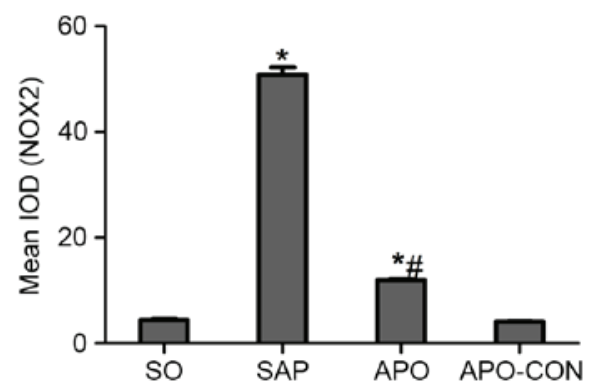

C

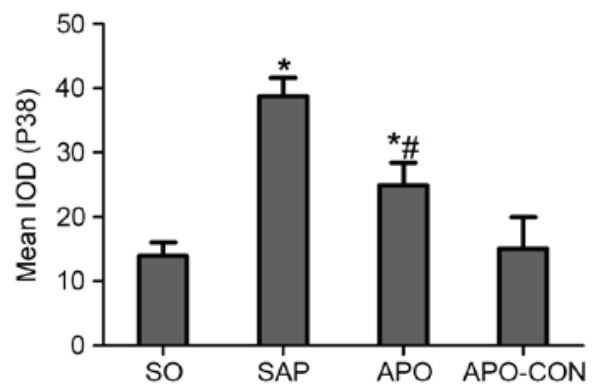

B

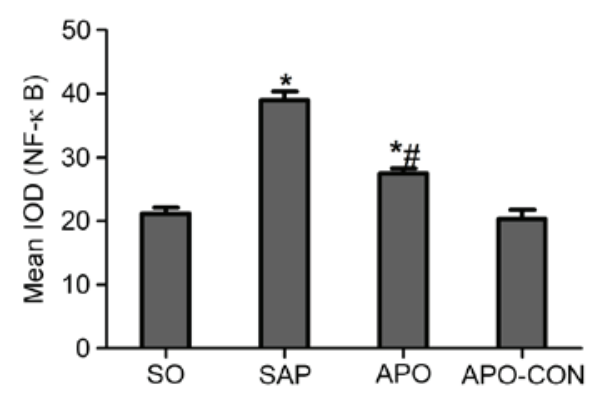

D

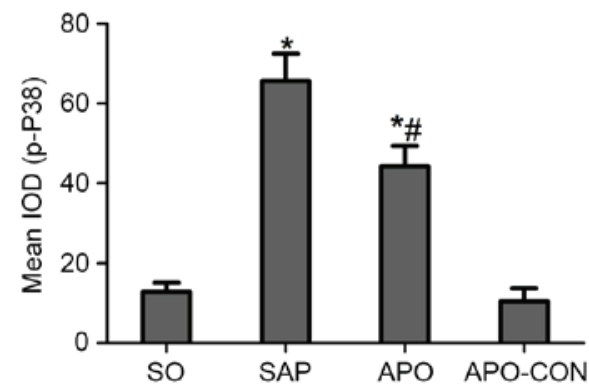

Figure 9. Immunohistochemical analysis of (A) nicotinamide adenine dinucleotide phosphate oxidase 2 (NOX2); (B) nuclear factor (NF)-кB; (C) p38 mitogen-activated protein kinases (P38); (D) phosphorylated (p)-P38. Results are presented as the mean \pm standard deviation. "P<0.05 vs. sham operation (SO) group; "P<0.05 vs. severe acute pancreatitis (SAP) group. APO-CON, drug control group; APO, apocynin group; IOD, integrated optical density.

cytoplasmic vesicles, including endoplasmic reticulum and mitochondria, were characteristics observed at a magnification of $\mathrm{x} 2,500$ (Fig. 10C). The integrity of the microvilli and IECs of rats in the APO group was significantly improved (Fig. 10D). The ultrastructural damage to mitochondria, endoplasmic reticulum, ribosomes and other organelles was ameliorated by apocynin treatment (Fig. 10D). Ultrastructural changes in the APO-CON group were similar to the SO group.

\section{Discussion}

NOX is a ROS-generating transmembrane flavoprotein enzyme, which has a key role in innate immunity, mitogenic signaling, hormone synthesis, apoptosis, angiogenesis, and oxidative modification of the extracellular matrix and extracellular proteins (8). The involvement of NOX in the pathophysiology of cerulean-induced pancreatitis has been 

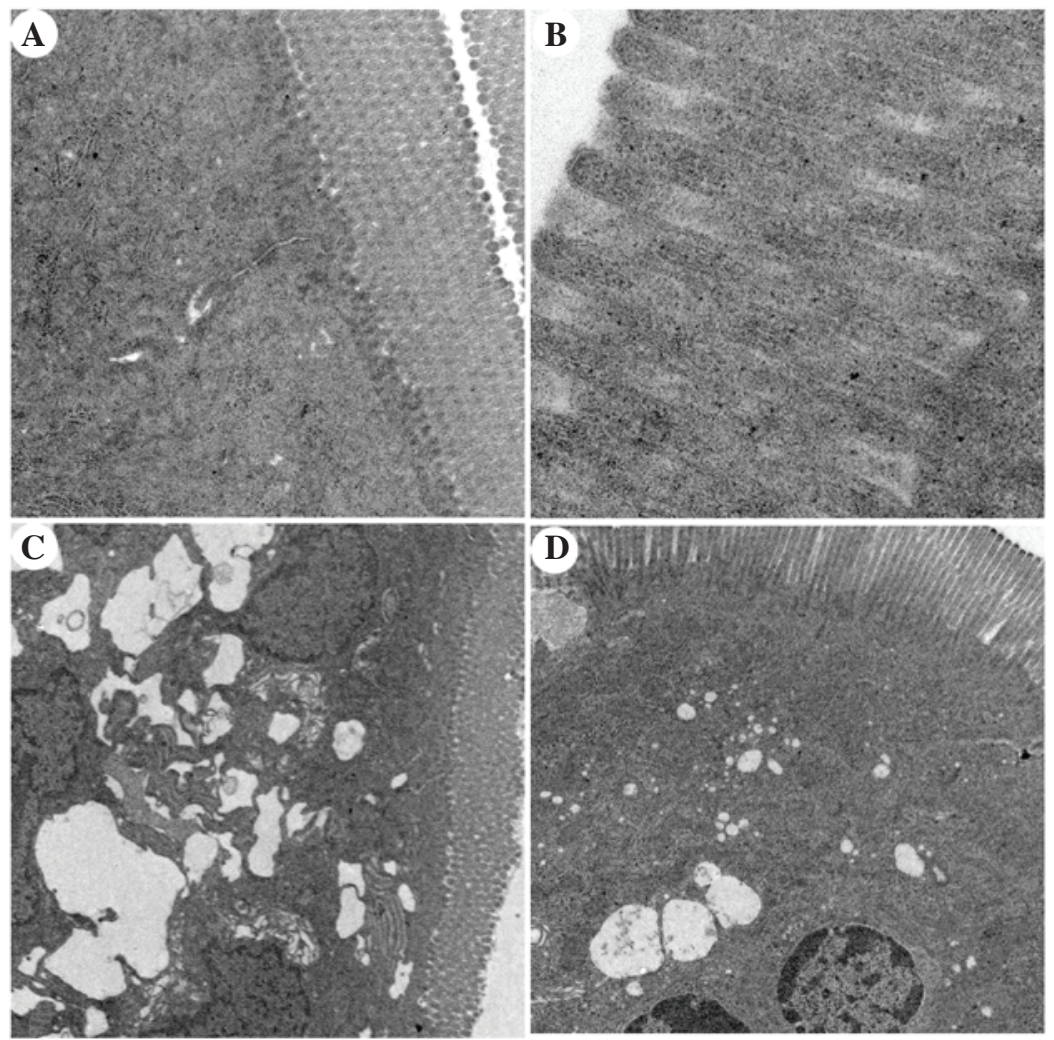

Figure 10. Transmission electron microscopic comparison of intestinal epithelial architecture. (A) Intestinal epithelium of the sham operation (SO) group exhibited columnar intestinal epithelial cells (IECs) and long microvilli. Cytoplasmic organelles were normal (magnification, x5,000). (B) The integrity of microvilli in the SO group was maintained and microvilli were long (magnification, x20,000). (C) In the severe acute pancreatitis (SAP) group, IECs and microvilli were not intact, microvilli were absent and became shorter in various locations. The mitochondria and endoplasmic reticulum were grossly expanded and ribosome numbers were significantly increased. The nuclear double membrane appeared irregular in shape (magnification, x2,500). (D) In the apocynin group, IECs were intact and microvilli became longer than in the SAP group. Enlarged mitochondria, endoplasmic reticulum and ribosomes were significantly decreased compared with the SAP group (magnification, x2,500).

indicated by increased NOX activity in AR42 $\mathrm{J}$ cells 15 min after induction of experimental pancreatitis, accompanied by NF- $\kappa$ B activation and IL-6 expression (10). In addition, the involvement of NOX has been studied in several models of acute pancreatitis and has been recognized to contribute to its progression (7,16-18). Pancreatitis-induced release of inflammatory factors, cytokines and growth factors may be responsible for local and distant organ NOX activation (19), thus resulting in further development of acute pancreatitis. It has previously been reported that apocynin treatment reduces serum levels of TNF- $\alpha$, IL- $1 \beta$ and NF- $\kappa B$, and intercellular adhesion molecule 1 expression, and decreases the severity of acute lung inflammation (20). Apocynin has also been used in several experimental studies, including those associated with liver injury (21), lung injury (22), kidney injury (23), brain injury (24) and ischemic reperfusion injury $(25,26)$. The results of the present study further supported the beneficial effects of treatment with the NOX antagonist apocynin, since the severity of pancreatitis-associated intestinal barrier dysfunction was ameliorated following apocynin treatment.

Pancreatitis-associated gut barrier dysfunction is characterized by the passage of enteric bacteria through the mucosal barrier to extraintestinal sites (bacterial translocation), impaired intestinal motility, and increased intestinal permeability (27). Destruction of the intestinal mucosal mechanical barrier also results in the release of a large amount of DAO enzymes and active substances (28). Previous studies have reported that intestinal barrier injury may lead to SIRS and MODS (29,30). DAO is an effective biomarker, which reflects the integrity and mucosal function of the small intestine. The concentration of DAO in the serum and mucosa of the small intestine may be determined as a measure of small intestine barrier function. In the present study, TEM demonstrated that microvilli of the intestinal mucosa exhibited reduced width and height, tight junctions were damaged and DAO levels were increased in rats in the SAP group. These parameters were significantly reduced following apocynin treatment.

The present study demonstrated that SAP was successfully induced in the rats after $12 \mathrm{~h}$ of modeling, as characterized by increased serum levels of AMY and LIP, pancreatic tissue injury, and pathological alterations. The serum levels of DAO combined with negative morphological alterations to the ileum indicated the presence of obvious intestinal barrier dysfunction and tissue injury during the progression of SAP. The results demonstrated that pretreatment with apocynin attenuated the following: i) Serum AMY and LIP levels; ii) serum DAO levels; iii) morphological alterations to the pancreas and intestine; iv) proinflammatory cytokine production; v) MDA and SOD content; vi) NOX2, NF- $\mathrm{kB}$ and p38 MAPK expression; and vii) ultrastructural alterations to the intestinal mucosa. All of these observations suggested that NOX may participate in the process of intestinal barrier 
damage in SAP, and treatment with a NOX inhibitor exerts potent anti-inflammatory and antioxidative effects, and ameliorates the degree of SAP and associated intestinal barrier injury in rats.

It has been demonstrated that ROS generated by NOX have prominent roles in the pathogenesis of acute pancreatitis (31). Under physiological conditions, tissues contain various endogenous antioxidant enzymes, including glutathione and SOD, which scavenge ROS and prevent lipid peroxidation (32). In the process of SAP, ROS are overproduced, thus inducing an imbalance between ROS and endogenous antioxidants or antioxidant enzymes. The overproduced ROS are able to directly or indirectly damage intestinal tissues, resulting in intestinal barrier dysfunction and histological alterations. The results of the present study demonstrated that SOD activity was significantly increased following apocynin treatment of SAP rats. Conversely, MDA levels were decreased. These results, combined with the intestinal morphological alterations detected, indicated that apocynin may reduce ROS levels by inhibiting NOX, further ameliorating intestinal oxidative damage.

NOX is a major contributor during inflammation, and has a role as an inflammatory stimulator, activating the leukocyte system, which can then produce and release various secondary inflammatory mediators. It has previously been demonstrated that apocynin significantly inhibits the expression levels of TNF- $\alpha$ and IL-1 $\beta$, which are potent triggers associated with leukocyte migration, and that suppression of NOX activity by apocynin results in attenuation of ROS-mediated signal transduction and inducible nitric oxide synthase expression in lipopolysaccharide + interferon $\gamma$-stimulated microvascular endothelial cells (20). In addition, evidence suggests that inflammatory cell infiltration, including infiltration of TNF- $\alpha$, IL-1 $\beta$ and IL-6, is an early and vital event in acute pancreatitis, which leads to local and systemic complications (19). Data from the present study demonstrated that these cytokines were increased following induction of SAP; however, their serum levels were reduced in rats treated with apocynin. In addition, in the SAP group, intestinal barrier dysfunction was correlated with increased intestinal injury, as confirmed using histological analysis. In the APO group, milder swelling, necrosis and loss of mucosal structure, and reduced inflammatory cell infiltration was detected compared with in the SAP group. Pathological grading of intestinal injury was significantly decreased in the SAP group pretreated with APO.

ROS are able to induce cytokine expression, apoptosis, and NF- $\mathrm{B}, \mathrm{MAPK}$ and Janus kinase/signal transducer and activator of transcription pathway activation, thus regulating inflammation and apoptosis in pancreatic acinar cells (19). Consequently, NOX, NF- $\mathrm{B}$ and MAPK may be involved in the pathogenesis of acute pancreatitis (33). NF- $\kappa \mathrm{B}$ is a transcription factor that is necessary for the transcription of numerous proinflammatory mediators, including cytokines, chemokines, and oxygen-derived free radicals. In quiescent cells, NF- $\kappa \mathrm{B}$ is present in the cytosol where it is complexed with $I \kappa B$. The phosphorylation of $I \kappa B$ on serines within the amino-terminal domain results in the dissociation of $N F-\kappa B$ and its subsequent translocation to the nucleus, consequently initiating gene transcription (34). Translocation of NF- $\kappa \mathrm{B}$ to the nucleus mediates the expression of cytokines, which has a major role in the pathogenesis of pancreatitis (33). It has previously been reported that inhibition of $\mathrm{NF}-\kappa \mathrm{B}$ activation efficiently suppresses IL-6 expression and attenuates the severity of cerulean-induced pancreatitis in AR42J cells (10). The present study demonstrated that the translocation of activated $\mathrm{NF}-\kappa \mathrm{B}$ into the nucleus was significantly increased in the intestinal tissues of SAP rat. However, following treatment with apocynin, the nuclear expression of $\mathrm{NF}-\kappa \mathrm{B}$ was decreased. These results suggested that the activation of $\mathrm{NF}-\kappa \mathrm{B}$ could be inhibited by apocynin via NOX suppression.

P38 MAPK is serine-threonine kinase, which mediates intracellular signaling associated with several cellular activities, including cell proliferation, differentiation, survival, death and transformation (35). Recently, it has been revealed that p38 MAPK may be involved in the NOX signal transduction pathway. ROS mediate activation of the p38 MAPK pathway and promote protein phosphorylation in pancreatic acinar cells (19). A previous study reported that p38 MAPK is associated with the severity of pancreatitis and in the respiratory distress syndrome associated with acute pancreatitis (34). Another study using pancreatic fragments stimulated by the cholecystokinin analog, cerulein, demonstrated that proinflammatory mediator production was increased in parallel with the activation of p38 MAPK, and that these alterations were attenuated by treatment with chemical inhibitors of p38 MAPK (36).

During the progression of SAP, the p38 MAPK signaling pathway is involved in the regulation of NF- $\kappa \mathrm{B}$ activation, which has a crucial role in the inflammatory cascade $(37,38)$. Activation of p38 MAPK results in phosphorylation of MAPK-activated protein kinase 2, a downstream protein kinase of p38 MAPK, which triggers NF- $\kappa \mathrm{B}$ and exaggerates inflammation (39). The present study demonstrated that intestinal p38 and p-p38 expression in the SAP group was markedly increased. Conversely, p38 expression was suppressed by apocynin pretreatment, thus suggesting that p38 MAPK may be involved in the pathogenesis of intestinal injury in SAP rats. These results suggested that the NOX inhibitor apocynin may inhibit the expressions of the inflammatory cytokines by suppressing NF- $\kappa \mathrm{B}$ activation and $\mathrm{p} 38$ expression. The results also indicated that intestinal cells constitutively expressed the NOX subunit NOX2.

In conclusion, SAP-associated intestinal barrier dysfunction was aggravated in the SAP group. Treatment with apocynin, a NOX inhibitor, reduced the severity of pancreatitis-associated intestinal dysfunction, which was associated with a reduction in the systemic concentration of cytokines, reduced oxidative stress and downregulated NF- $\kappa \mathrm{B}$ and p38 MAPK expression. Thus, apocynin treatment may ameliorate SAP-induced intestinal barrier injury and these findings may provide a basis for further clinical studies of apocynin as a novel and adjuvant therapy to treat intestinal barrier injury with SAP.

\section{Acknowledgements}

The present study was supported by the NSFC (grant no. 81360081/H0320), the Natural Science Foundation of Hubei Province (grant no. 2013CFB459), and the Independent Research Project of Wuhan University (grant no. 2042015KF0090). 


\section{References}

1. Lankisch PG, Apte M and Banks PA: Acute pancreatitis. Lancet 386: 85-96, 2015.

2. Ammori BJ: Role of the gut in the course of severe acute pancreatitis. Pancreas 26: 122-129, 2003.

3. Rahman SH, Ammori BJ, Holmfield J, Larvin $M$ and McMahon MJ: Intestinal hypoperfusion contributes to gut barrier failure in severe acute pancreatitis. J Gastrointest Surg 7: 26-35; discussion 35-36, 2003.

4. Capurso G, Zerboni G, Signoretti M, Valente R, Stigliano S, Piciucchi M and Delle Fave G: Role of the gut barrier in acute pancreatitis. J Clin Gastroenterol 46: S46-S51, 2012.

5. Zhang XP, Zhang J, Song QL and Chen HQ: Mechanism of acute pancreatitis complicated with injury of intestinal mucosa barrier J Zhejiang Univ Sci B 8: 888-895, 2007.

6. Tian R, Tan JT, Wang RL, Xie H, Qian YB and Yu KL: The role of intestinal mucosa oxidative stress in gut barrier dysfunction of severe acute pancreatitis. Eur Rev Med Pharmacol Sci 17: 349-355, 2013.

7. Masamune A, Watanabe T, Kikuta K, Satoh K and Shimosegawa T: NADPH oxidase plays a crucial role in the activation of pancreatic stellate cells. Am J Physiol Gastrointest Liver Physiol 294: G99-G108, 2008.

8. Lambeth JD: NOX enzymes and the biology of reactive oxygen. Nat Rev Immunol 4: 181-189, 2004.

9. Hancock JT, Desikan R and Neill SJ: Hydrogen peroxide and nitric oxide in plant defence: Revealing potential targets for oxidative stress tolerance? Biofactors 15: 99-101, 2001.

10. Yu JH, Lim JW, Kim H and Kim KH: NADPH oxidase mediates interleukin-6 expression in cerulein-stimulated pancreatic acinar cells. Int J Biochem Cell Biol 37: 1458-1469, 2005.

11. Gukovskaya AS, Vaquero E, Zaninovic V, Gorelick FS, Lusis AJ, Brennan ML, Holland S and Pandol SJ: Neutrophils and NADPH oxidase mediate intrapancreatic trypsin activation in murine experimental acute pancreatitis. Gastroenterology 122: 974-984, 2002.

12. Uysal A, Sahna E, Ozguler IM, Burma O and Ilhan N: Effects of apocynin, an NADPH oxidase inhibitor, on levels of ADMA, MPO, iNOS and TLR4 induced by myocardial ischemia reperfusion. Perfusion 30: 472-477, 2015.

13. Zhang YS, He L, Liu B, Li NS, Luo XJ, Hu CP, Ma QL, Zhang GG, Li YJ and Peng J: A novel pathway of NADPH oxidase/vascular peroxidase 1 in mediating oxidative injury following ischemia-reperfusion. Basic Res Cardiol 107: 266, 2012.

14. Schmidt J, Rattner DW, Lewandrowski K, Compton CC, Mandavilli U, Knoefel WT and Warshaw AL: A better model of acute pancreatitis for evaluating therapy. Ann Surg 215: 44-56, 1992.

15. Chiu CJ, McArdle AH, Brown R, Scott HJ and Gurd FN: Intestinal mucosal lesion in low-flow states. I. A morphological, hemodynamic, and metabolic reappraisal. Arch Surg 101: 478-483, 1970

16. Mohammed AM, Syeda K, Hadden T and Kowluru A: Upregulation of phagocyte-like NADPH oxidase by cytokines in pancreatic beta-cells: Attenuation of oxidative and nitrosative stress by 2-bromopalmitate. Biochem Pharmacol 85: 109-114, 2013.

17. Rebelato E, Mares-Guia TR, Graciano MF, Labriola L, Britto LR, Garay-Malpartida HM, Curi R, Sogayar MC and Carpinelli AR: Expression of NADPH oxidase in human pancreatic islets. Life Sci 91: 244-249, 2012.

18. Hu R, Wang YL, Edderkaoui M, Lugea A, Apte MV and Pandol SJ: Ethanol augments PDGF-induced NADPH oxidase activity and proliferation in rat pancreatic stellate cells. Pancreatology 7: 332-340, 2007.

19. Cao WL, Xiang XH, Chen K, Xu W and Xia SH: Potential role of NADPH oxidase in pathogenesis of pancreatitis. World J Gastrointest Pathophysiol 5: 169-177, 2014.

20. Impellizzeri D, Esposito E, Mazzon E, Paterniti I, Di Paola R, Bramanti P and Cuzzocrea S: Effect of apocynin, a NADPH oxidase inhibitor, on acute lung inflammation. Biochem Pharmacol 81: 636-648, 2011.
21. Kono H, Rusyn I, Uesugi T, Yamashina S, Connor HD, Dikalova A, Mason RP and Thurman RG: Diphenyleneiodonium sulfate, an NADPH oxidase inhibitor, prevents early alcohol-induced liver injury in the rat. Am J Physiol Gastrointest Liver Physiol 280: G1005-G1012, 2001.

22. Dodd-O JM and Pearse DB: Effect of the NADPH oxidase inhibitor apocynin on ischemia-reperfusion lung injury. Am J Physiol Heart Circ Physiol 279: H303-H312, 2000.

23. Joshi S, Peck AB and Khan SR: NADPH oxidase as a therapeutic target for oxalate induced injury in kidneys. Oxid Med Cell Longev 2013: 462361, 2013.

24. Ferreira AP, Rodrigues FS, Della-Pace ID, Mota BC, Oliveira SM, Velho Gewehr Cde C, Bobinski F, de Oliveira CV, Brum JS, Oliveira MS, et al: The effect of NADPH-oxidase inhibitor apocynin on cognitive impairment induced by moderate lateral fluid percussion injury: Role of inflammatory and oxidative brain damage. Neurochem Int 63: 583-593, 2013.

25. Genovese T, Mazzon E, Paterniti I, Esposito E, Bramanti P and Cuzzocrea S: Modulation of NADPH oxidase activation in cerebral ischemia/reperfusion injury in rats. Brain Res 1372: 92-102, 2011.

26. Liu PG, He SQ, Zhang YH and Wu J: Protective effects of apocynin and allopurinol on ischemia/reperfusion-induced liver injury in mice. World J Gastroenterol 14: 2832-2837, 2008.

27. Leveau P, Wang X, Sun Z, Börjesson A, Andersson E and Andersson R: Severity of pancreatitis-associated gut barrier dysfunction is reduced following treatment with the PAF inhibitor lexipafant. Biochem Pharmacol 69: 1325-1331, 2005.

28. Zhang JW, Zhang GX, Chen HL, Liu GL, Owusu L, Wang YX, Wang GY and Xu CM: Therapeutic effect of Qingyi decoction in severe acute pancreatitis-induced intestinal barrier injury. World J Gastroenterol 21: 3537-3546, 2015.

29. Sun JJ, Chu ZJ, Liu WF, Qi SF, Yang YH, Ge PL, Zhang XH, Li WS, Yang C and Zhang YM: Perirenal space blocking restores gastrointestinal function in patients with severe acute pancreatitis. World J Gastroenterol 19: 8752-8757, 2013

30. Yue C, Wang W, Tian WL, Huang Q, Zhao RS, Zhao YZ, Li QR and Li JS: Lipopolysaccharide-induced failure of the gut barrier is site-specific and inhibitable by growth hormone. Inflamm Res 62: 407-415, 2013

31. Leung PS and Chan YC: Role of oxidative stress in pancreatic inflammation. Antioxid Redox Signal 11: 135-165, 2009.

32. Ju KD, Lim JW, Kim KH and Kim H: Potential role of NADPH oxidase-mediated activation of Jak2/Stat 3 and mitogen-activated protein kinases and expression of TGF- $\beta 1$ in the pathophysiology of acute pancreatitis. Inflamm Res 60: 791-800, 2011.

33. Kim H: Cerulein pancreatitis: Oxidative stress, inflammation, and apoptosis. Gut Liver 2: 74-80, 2008.

34. Williard DE, Twait E, Yuan Z, Carter AB and Samuel I: Nuclear factor kappa B-dependent gene transcription in cholecystokininand tumor necrosis factor-alpha-stimulated isolated acinar cells is regulated by p38 mitogen-activated protein kinase. Am J Surg 200: 283-290, 2010.

35. Yang Y, Kim SC, Yu T, Yi YS, Rhee MH, Sung GH, Yoo BC and Cho JY: Functional roles of p38 mitogen-activated protein kinase in macrophage-mediated inflammatory responses. Mediators Inflamm 2014: 352371, 2014

36. Cao MH, Xu J, Cai HD, Lv ZW, Feng YJ, Li K, Chen CQ and Li YY: p38 MAPK inhibition alleviates experimental acute pancreatitis in mice. Hepatobiliary Pancreat Dis Int 14: 101-106, 2015.

37. Papachristou DJ, Papadakou E, Basdra EK, Baltopoulos P, Panagiotopoulos E and Papavassiliou AG: Involvement of the p38 MAPK-NF-kappaB signal transduction pathway and COX-2 in the pathobiology of meniscus degeneration in humans. Mol Med 14: 160-166, 2008.

38. Zhu L, Song S, Pi Y, Yu Y, She W, Ye H, Su Y and Hu Q: Cumulated $\mathrm{Ca}^{+}$spike duration underlies $\mathrm{Ca}^{+}$oscillation frequency-regulated $\mathrm{NF} \kappa \mathrm{B}$ transcriptional activity. J Cell Sci 124: 2591-2601, 2011

39. Tudhope SJ, Finney-Hayward TK, Nicholson AG, Mayer RJ, Barnette MS, Barnes PJ and Donnelly LE: Different mitogen-activated protein kinase-dependent cytokine responses in cells of the monocyte lineage. J Pharmacol Exp Ther 324: 306-312, 2008 . 